\title{
Knockdown of EPHA1 Using CRISPR/CAS9 Suppresses Aggressive Properties of Ovarian Cancer Cells
}

\author{
YUXIN CUI ${ }^{1 *}$, BO WU ${ }^{1,2^{*}}$, VALENTINA FLAMINI ${ }^{1}$, BRONWEN A.J. EVANS $^{1,3}$, \\ DESHAN ZHOU ${ }^{2}$ and WEN G. JIANG ${ }^{1}$ \\ ${ }^{1}$ Cardiff China Medical Research Collaborative, Cardiff University School of Medicine, Cardiff, U.K.; \\ ${ }^{2}$ Department of Histology and Embryolog, Cancer Institute, \\ Key Laboratory of Cancer Metastasis (Beijing), Capital Medical University, Beijing, P.R. China; \\ ${ }^{3}$ Institute of Molecular and Experimental Medicine, School of Medicine, Cardiff University, Cardiff, U.K.
}

\begin{abstract}
Background/Aim: Overexpression of erythropoietinproducing hepatocellular Al (EPHAl), a member of the EPH super family, is frequently observed in various cancer types. The dysregulated interaction of EPHAl with its ligand Ephrin Al has been linked to the progression of ovarian cancer $(O C)$. However, the contribution of EPHAl in the regulation of the aggressive properties of $O C$ cells remains unknown. Materials and Methods: In this study we investigated the differential expression of EPHAI in human OC cells. The EPHAl gene was knocked-down using the CRISPR/Cas 9 technique to evaluate its effect on the progressive properties of OC cells. Results: After EPHAl was knocked-down using a CRISPR/CAS9 genomic editing system in OC cells (SKOV3 and COV504), we observed cellcycle arrest at the $G_{0} / G_{1}$ phases in both OC cell lines. Knockdown of EPHAI in the two OC cells inhibited their aggressive traits, including proliferation, invasion and migration, as well as improving their attachment to extracellular matrix. EPHAl may play a role in OC through its regulation of multiple signaling pathways, such as matrix metalloproteinase-2 (MMP2), extracellular signal-regulated kinase 2 (ERK2) and proto-oncogene c-MYC. Conclusion: EPHA1 may promote the aggression of some OC cells and, thus, be considered a potential therapeutic target for the treatment of malignant $O C$.
\end{abstract}

*These Authors contributed equally to this study.

Correspondence to: Professor Wen G. Jiang, Cardiff China Medical Research Collaborative, Henry Wellcome Building, School of Medicine, Cardiff University, Heath Park. Cardiff, CF14 4XN, U.K Tel: +4402920687065, e-mail: jiangw@cf.ac.uk

Key Words: EPHA1, CRISPR, CAS9, ovarian carcinoma cells, adhesion, migration.
Ovarian cancer (OC) is the seventh most common type of cancer and cancer-related death in women worldwide and contributes to approximately $4 \%$ of all female cancers (1). Although the lifetime risk of OC in women is $1.5 \%$, it is considered as the most deadly of gynecological cancers (2). As OC is often asymptomatic in the early stages, it is often diagnosed when the disease is in the later stages (III and IV). The 5-year survival rate for the women diagnosed with OC at an advanced stage is approximately $19 \%$, despite the $90 \%$ rate for patients with early diagnosis (3). However, the diagnosis techniques of $\mathrm{OC}$ at its early stages are not well developed and lack accuracy (4). Currently, the etiology of OC is not fully understood despite varied proposed pathogenic hypotheses $(5,6)$.

With 14 members, the erythropoietin-producing hepatocellular (EPH) receptors represent the largest subfamily of the receptor tyrosine kinases $(7,8)$. EPH receptors are categorized into two subfamilies, EPHA and EPHB, based on their sequence homology and preferential binding potential to their ephrin-A and ephrin-B ligands, respectively $(9,10)$. In humans, there is evidence to show expression of nine EPHA (EPHA1-8, 10) and five EPHB (EPHB1-4, 6) receptors, as well as five ephrin-A and three ephrin-B ligands (11). Following activation by the binding of their ephrin ligands, EPH receptors play a variety of roles in physiological and pathological processes, including immune cell development and behaviors, tumorigenesis, tumor-associated angiogenesis and tumor progression (12).

EPH receptor A1 (EPHA1) was originally isolated from erythropoietin-producing hepatocellular carcinoma cell lines (13). The over-expression of EPHA1 has been described in several human cancers, including colorectal (14), breast (15) and prostate (16). However, EPHA1 is found to be down-regulated in non-melanoma skin cancer (17) and glioblastoma. In OC, the expression profile of EPHA1 has not been well-documented. One previous study 
indicated that the overexpression of EPHA1 and its ligand Ephrin A1 may be correlated with shortened survival in patients with OC (18). Little is known whether EPHA1 plays a role in the aggressive properties of OC cells. In this study, we assessed the expression of EPHA1 in OC cells and attempted to investigate the effect of EPHAl knockdown (KD), using the CRISPR/Cas9 technique on two OC cell line behaviors.

\section{Materials and Methods}

Cell culture. Human OC cell lines, SKOV3 and COV504, were purchased from American Type Culture Collection (ATCC, Rockville, MD, USA). COV504 cells were cultured in DMEM supplemented with $10 \%$ fetal bovine serum (Invitrogen, Paisley, UK) and $1 \%$ penicillin/streptomycin solution (Invitrogen). SKOV3 cells were maintained in McCoys 5A medium supplemented with $10 \%$ fetal bovine serum (Invitrogen) and 1\% penicillin/streptomycin solution (Invitrogen). Both cell lines were cultured at $37^{\circ} \mathrm{C}$ under a humidified atmosphere of $5 \% \mathrm{CO}_{2}$.

RNA extraction and reverse transcription-polymerase chain reaction (RT-PCR). Total RNA was extracted from cultured cells using TRI reagent (Sigma-Aldrich, Irvine, UK) according to the manufacturer's instructions and quantified using a Nanodrop spectrophotometer. cDNA samples were generated from the total RNA using GoScript ${ }^{\mathrm{TM}}$ Reverse Transcription System kit (Promega, Madison, WI, USA). Subsequently, PCR was conducted using a REDTaq ${ }^{\mathrm{TM}}$ ReadyMix PCR reaction mix (Sigma-Aldrich) on a 2720 Thermo Cycler (Applied Biosystems, Warrington, UK). The following primers were used: EPHA1: forward, 5'-CCTTATGCCAACTACACCTT-3' and reverse, 5'-GTTCTTCATCCTGGTTCAGC-3'. GAPDH: forward, 5'GGCTGCTTTTAACTCTGGTA-3' and reverse, 5'-ACTGTGGTCAT GAGTCCTT-3'. The following cycling conditions were used: $94^{\circ} \mathrm{C}$ for $5 \mathrm{~min}, 30$ cycles of $94^{\circ} \mathrm{C}$ for $30 \mathrm{~s}, 55^{\circ} \mathrm{C}$ for $30 \mathrm{~s}$ and $72^{\circ} \mathrm{C}$ for 30 s, followed by $72^{\circ} \mathrm{C}$ for $10 \mathrm{~min}$. PCR products were separated on a 1 $\%$ agarose gel and photographed using a VisiDoc-It imaging system (Ultra-Violet Products Ltd., San Gabriel, CA, USA).

EPHA1-specific CRISPR/CAS9 vectors and stable cell line establishment. Construction of CRISPR/Cas9 vectors that targeted EPHA1 have been described in one of our previous studies (19). Briefly, human CRISPR/CAS9 guide RNA primers were designed based on the gene EPHAl (GenBank accession number: NM_005232). The primer sequences for were; EPHAl casF1 (guide s): 5'CACCGCCTGGGGCTGGCCCGCGCGT 3', EPHA1 casR1 (guide as): 5'AAACACGCGCGGGCCAGCCCCAG3'. The primer oligoes were annealed using the following parameters: $88^{\circ} \mathrm{C}$ for 2 $\min ; 65^{\circ} \mathrm{C}$ for $10 \mathrm{~min} ; 37^{\circ} \mathrm{C} 10 \mathrm{~min} ; 25^{\circ} \mathrm{C} 5 \mathrm{~min}$. The annealing primer was then purified and cloned into the pSpCas9 (BB)-2APuro (PX459) vector (Plasmid \#62988; Addgene, Cambridge, MA, USA). The plasmid containing CRISPR/CAS9-EPHA1 was verified by sequencing analysis. Then PX459 vector containing CRISPR/CAS9 backbone and CRISPR/CAS9-EPHA1-gRNA plasmids were transfected into the two OC cells respectively by electroporation using Gene pulser (Bio-Rad, Hercules, CA, USA). After three weeks of selection with $2 \mu \mathrm{g} / \mathrm{ml}$ puromycin, the verified transfectants were cultured in maintenance medium with $0.5 \mu \mathrm{g} / \mathrm{ml}$ puromycin.
Immunofluorescence of EPHA1. Cells were seeded on chamber slides $\left(\mathrm{Nunc}^{\mathrm{TM}}\right)$ and incubated overnight. They were then fixed in $100 \%$ ethanol for $20 \mathrm{~min}$ and then washed with Hank's Balanced Salt Solution (HBSS). Triton X100 (0.3\%) was used to permeabilize cells before staining. Anti-EPHA1 antibody (\#sc-925; Santa Cruz Biotechnology, Santa Cruz, CA, USA) was incubated overnight at $4^{\circ} \mathrm{C}$. The slides were then incubated with a specific FITC labelled secondary antibody (Dako, Glostrup, Denmark) for $1 \mathrm{~h}$ at room temperature. Fluorescent staining was visualized and captured using an Olympus ${ }^{\mathrm{TM}}$ BX51 microscope equipped with a cooled C4742-80 digital camera (Hamamatsu Photonics, Enfield, UK).

Kinexus antibody microarray. Cultured cells were washed twice in ice-cold PBS and then sonicated in standard Kinexus lysis buffer (20 mm MOPS, 1\% Triton X-100, 2 mM EGTA, $5 \mathrm{mM}$ EDTA, $30 \mathrm{mM} \mathrm{NaF}, 60 \mathrm{mM} \beta$-glycerophosphate, $20 \mathrm{mM}$ sodium pyrophosphate, $1 \mathrm{~mm} \mathrm{Na} \mathrm{VO}_{4}, 1 \mathrm{mM}$ phenylmethylsulfonyl fluoride, $1 \times$ complete protease inhibitor mixture (Roche Applied Science, Mannheim, Germany), $1 \mathrm{mM}$ DTT). The protein concentration was then quantified with Fluorescamine protein dye (Sigma-Aldrich) using bovine serum albumin (BSA) as a standard. Approximately $100 \mu \mathrm{g}$ of lysate protein samples were shipped in dry ice to Kinexus Bioinformatics Corporation (Vancouver, BC, Canada) for Kinexus ${ }^{\mathrm{TM}}$ KAM-880 Antibody Microarray.

Cell cycle analysis. Detached cells were re-suspended in $1 \mathrm{ml}$ of ice cold $70 \% \mathrm{v} / \mathrm{v}$ ethanol and left on ice for $15 \mathrm{~min}$. Fixed cells were then centrifuged at $600 \times g$ for $5 \mathrm{~min}$, washed once with PBS. The pellets were then re-suspended in $500 \mu \mathrm{l}$ cell cycle staining solution in PBS $(50 \mu \mathrm{g} / \mathrm{ml}$ PI from $50 \mathrm{x}$ stock solution $(2.5 \mathrm{mg} / \mathrm{ml})$, $0.1 \mathrm{mg} / \mathrm{ml}$ RNase A and $0.05 \%$ Triton X-100), apart from the nonstained control. Cells were then incubated at room temperature in the dark for $15 \mathrm{~min}$. Following this, FACS analysis was performed on the BD FACSCanto ${ }^{\text {TM }}$ II flow cytometer (BD Biosciences, Oxford, UK) using the FL3 channel $(575 \mathrm{~nm})$. The FACS data were analyzed using the FCS Express v6.0 software (De Novo, Los Angeles, CA, USA).

In vitro cell-matrix adhesion. Cells (40,000 cells/well) were seeded into a 96-well plate pre-coated with Matrigel (50 $\mu \mathrm{l} /$ well) (BD Biosciences) and incubated at $37^{\circ} \mathrm{C}$ for 40 mins. Following a wash using PBS buffer, the cells were fixed with $4 \%$ formalin and stained with crystal violet. After capturing the images, the adherent cells were dissolved with $10 \%(\mathrm{v} / \mathrm{v})$ acetic acid and absorbance was determined using a plate reader.

In vitro wound healing assay. Approximately 40,000 cells were seeded into a 24-well plate and allowed to reach confluency. The cell monolayer was scraped using a P10 pipette tip. Photographs were taken at 0 and 4 hours after wounding. Migration distances were measured using the ImageJ software (National Institutes of Health, Bethesda, MD, USA).

In vitro transwell invasion and migration assay. A Transwell insert with $8 \mu \mathrm{m}$ pores was coated with $50 \mu \mathrm{g}$ of Matrigel for the invasion assay. Non-coated $8 \mu \mathrm{m}$ pores transwell inserts were used for the migration assay. Forty thousand cells were seeded into each insert for migration and invasion assay, respectively. After $72 \mathrm{~h}$ incubation, 1X Enzyme-Free Cell Dissociation Solution (Millipore, Billerica, MA, USA) with $2 \mu \mathrm{g} / \mathrm{ml}$ calcein AM (eBioscience, 
A

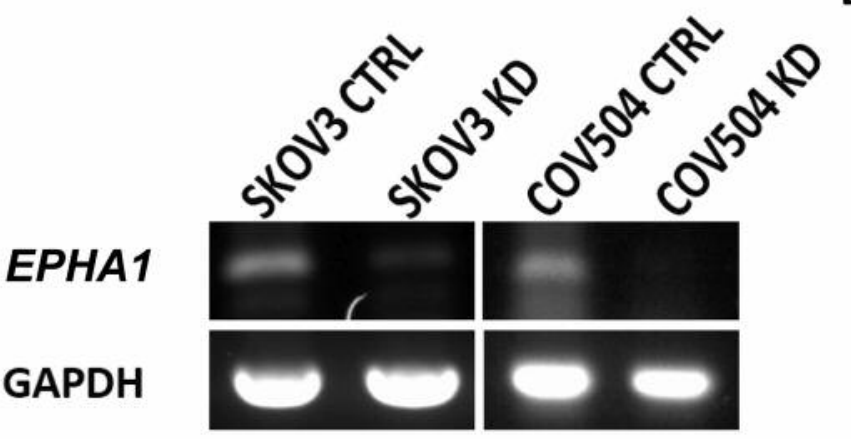

B

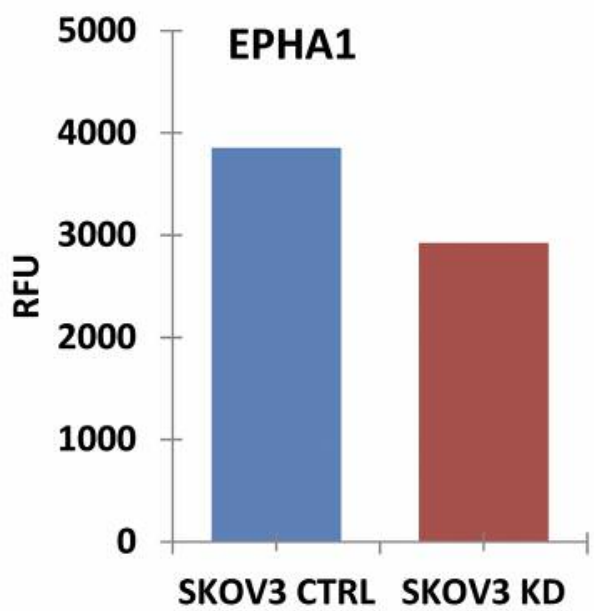

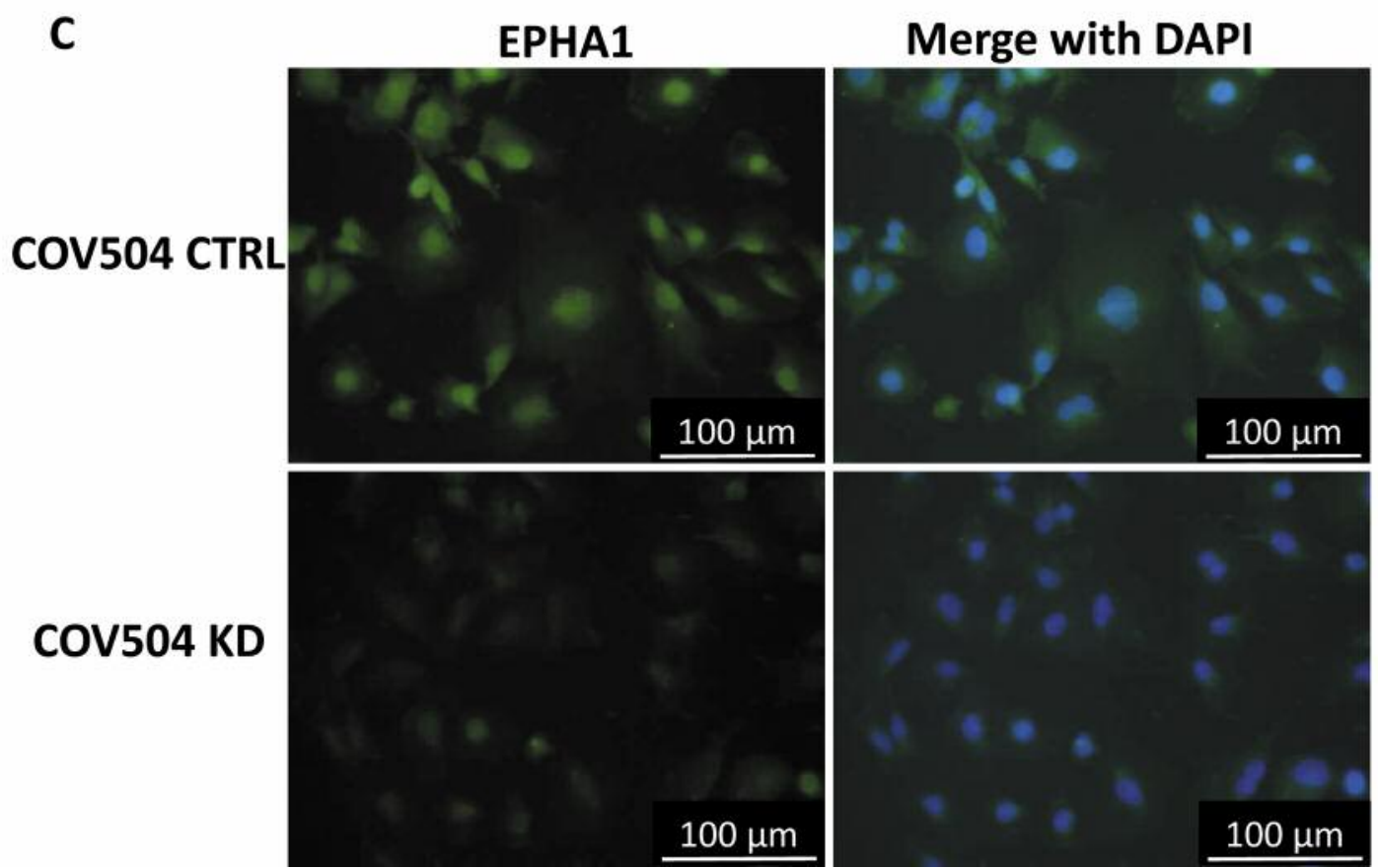

Figure 1. Evaluation of EPHA1 knock-down in two ovarian cancer $(O C)$ cells, SKOV3 and COV504. Stable cell lines were established using the EPHA1-gRNA CRISPR/Cas9 vectors (KD) and px459 backbone vector as a control (CTRL). (A) Gene expression of EPHAl as indicated by reverse transcription-polymerase chain reaction (RT-PCR) using GAPDH as house-keeping gene. (B) Protein expression of EPHA1 from two SKOV3 stable cells lines as indicated by the Kinex antibody array. (C) Immunofluorescence of EPHA1 in two COV504 stable cell lines using an EPHA1 specific antibody (Green) and nuclear counterstain DAPI (Blue).

Hatfield, UK) was added and the transwell inserts were incubated further for $1 \mathrm{~h}$ at $37^{\circ} \mathrm{C}$. Fluorescence was determined at a wavelength of ex490/em510 nm using the GloMax ${ }^{\circledR}$-Multi Detection System (Promega).

Electric cell-substrate impedance sensing (ECIS) system-based cell motility assay. The ECIS system (9600 model; Applied Biophysics Inc., Troy, NY, USA) was used to quantify cell migration. 96WIE arrays were used and cells were seeded at 40,000 cells per well in $200 \mu \mathrm{l}$ DMEM medium. The multi-frequency resistance was recorded using the ECIS system and an electric wound $(2,600 \mathrm{~Hz}$, $30 \mathrm{~s}$ ) was applied after monitoring for $15 \mathrm{~h}$. The data were analyzed using the ECIS-9600 software package.

Western blotting analysis. Western blot was performed as previously reported (20). Briefly, whole cell protein was extracted and quantified by NanoDrop 2000c spectrophotometer (Thermo Scientific, Waltham, MA, USA) using the BCA protein assay kit. After separation by SDSPAGE (a $10 \%$ sodium dodecyl sulfate polyacrylamide gel electrophoresis), the proteins were then transferred to a PVDF 

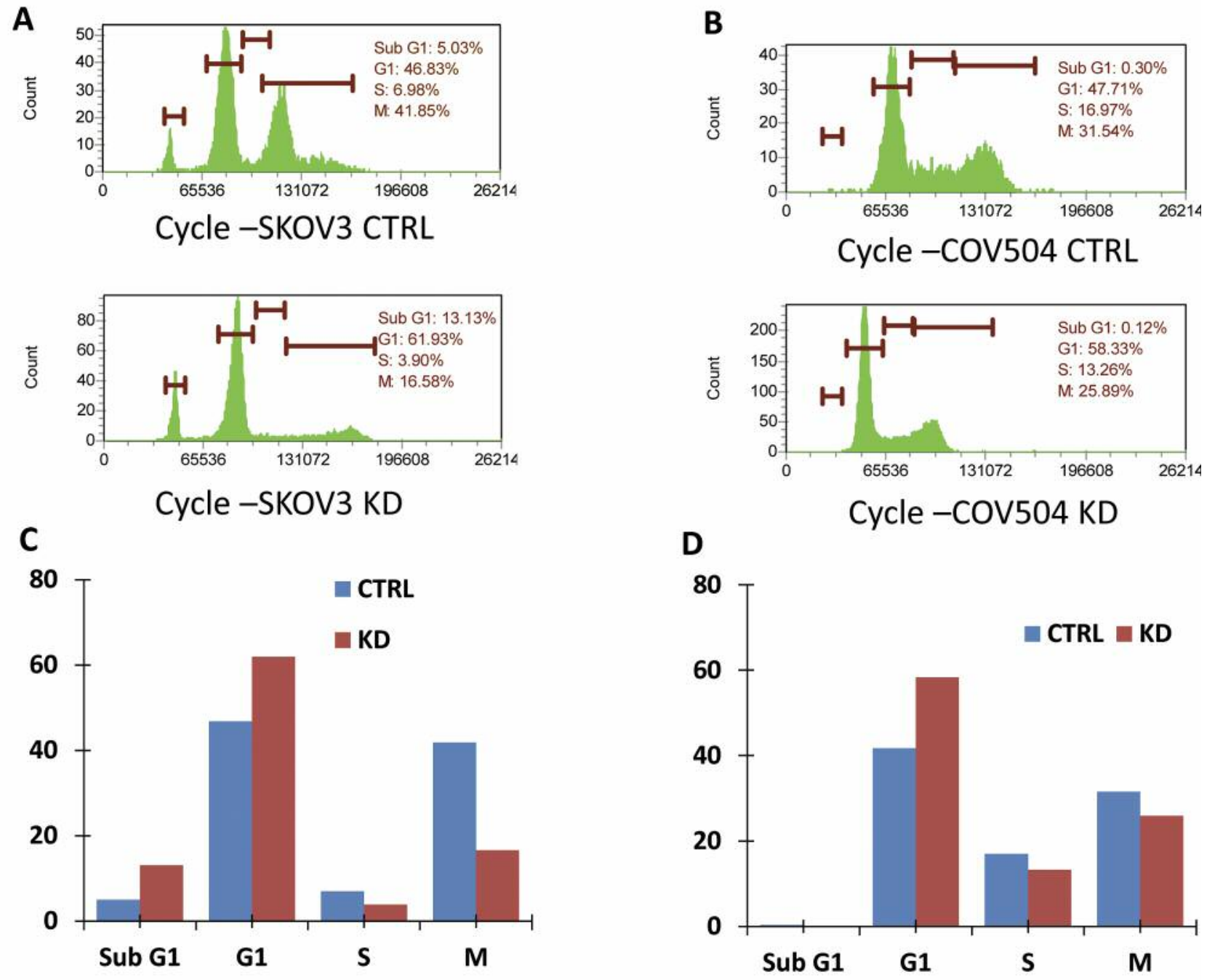

Figure 2. Cell-cycle analysis of the OC cells after EPHA1 KD. (A) and (C) Cell-cycle DNA profile and plot of the percentages of each phase from the SKOV3 stable cell lines, respectively. $(B)$ and $(D)$ Cell-cycle DNA profile and plot of the percentages of each phase from the COV504 stable cell lines, respectively. CTRL, Control; KD, knockdown; OC, ovarian cancer.

membrane (Millipore). Proteins were probed with primary antibodies anti-ERK or anti-JNK (1:500; Santa Cruz Biotechnology) and incubated with the corresponding horseradish peroxidase-conjugated secondary antibodies (1:2,000; Santa Cruz Biotechnology). The proteins were detected with Z-ECL chemiluminescence kit (Luminata Forte; Millipore, Hertfordshire, UK) and photographed using G-Box gel documentation system (Syngene, Cambridge, UK). GAPDH was used as an internal control. The intensity values were analyzed using the Image $\mathbf{J}$ software (NIH).

Statistical analysis. Statistical analysis was performed using SPSS18 (SPSS Inc., Chicago, IL, USA). The Student's $t$-test was used for data that were normally distributed. Patients' survival was analyzed using Kaplan-Meier analysis (21). The differences of the ECIS data were tested using the repeated measures analysis of variance (ANOVA). $p<0.05$ was considered statistically significant.

\section{Results}

Knockdown of EPHAl in OC cell lines. EPHAl gene expression was knocked down using the CRISPR/CAS9 method, targeting human EPHAl genomic DNA. The knockdown of EPHAl in the SKOV3 and COV504 OC cells was verified using PCR compared to their corresponding vector control cells (Figure 1A). The Kinexus protein array data also confirmed that there was reduction of the EPHA1 protein in SKOV3 cells when EPHAl was knocked-down (Figure 1B). Immunofluorescence cell staining also indicated a decrease of EPHA1 in COV504 cells (Figure 1C). Therefore, EPHAl can be knocked down in OC cells using the CRISP/Cas9 technique. 


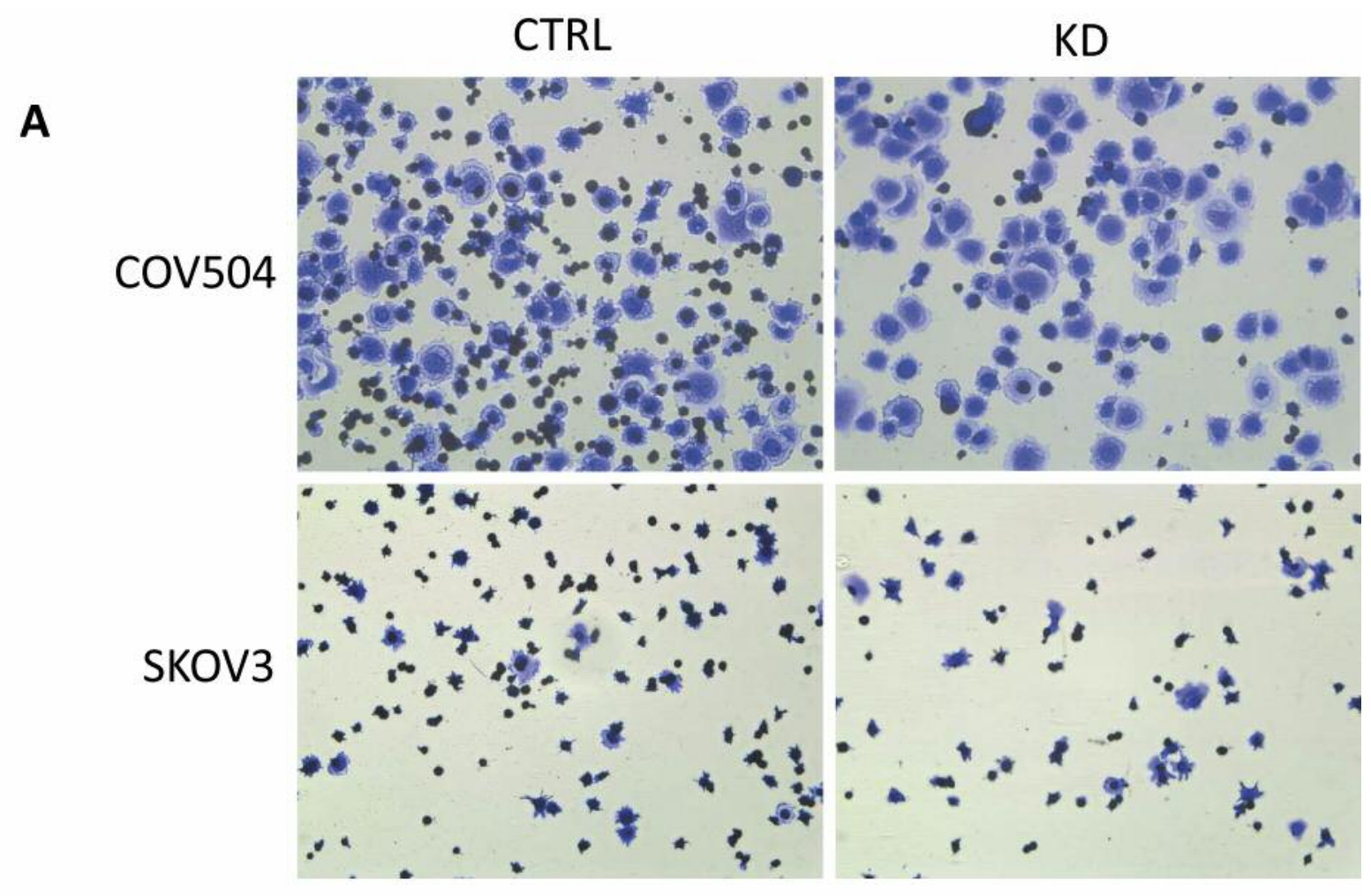

B

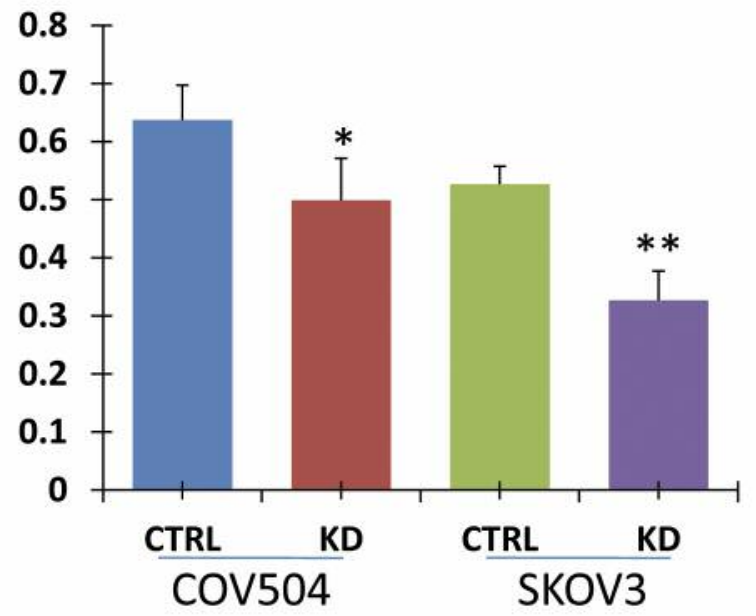

Figure 3. Adhesion to Matrigel of the OC cells after EPHA knockdown. (A) Representative images of the adhesive cells to Matrigel-coated surface that mimics extracellular matrix after $40 \mathrm{~min}$, which were stained crystal violet. (B) Quantification of the adhesive cells on Matrigel using a plate reader. Data are means with standard deviation (SD) (triple test, $n=3$ ). ${ }^{*} p<0.05 . * * p<0.01$. CTRL, Control; KD, knockdown; OC, ovarian cancer.

Knockdown of EPHAl induced $G_{0} / G_{1}$ cell cycle arrest in $O C$ cells. We evaluated the effect of EPHA1 on the cell cycle of OC cells by FACS. As shown in Figure 2, in the SKOV3 cells with EPHAl KD, there was increase in cells in the sub$\mathrm{G} 1$ (from $5.03 \%$ to $13.13 \%$ ) and $\mathrm{G}_{1}$ phases (from $46.83 \%$ to $61.93 \%)$, respectively, and a decrease of cells in the M phase ( $41.85 \%$ to $16.58 \%$ ) compared to the control with backbone vector (Figure 2A and C). In the COV504 cells, there was also increase in cells in the $G_{1}$ phase (from $47.71 \%$ to $58.33 \%$ ) and a decrease of cells in the M phase $(31.54 \%$ to $25.89 \%$ ) compared to the control with backbone vector (Figure 2B and D). The population of sub-G $\mathrm{G}_{1}$ phase COV504 cells was extremely low in the control cells $(0.30 \%)$ and did not change after EPHAl KD (0.12\%). 

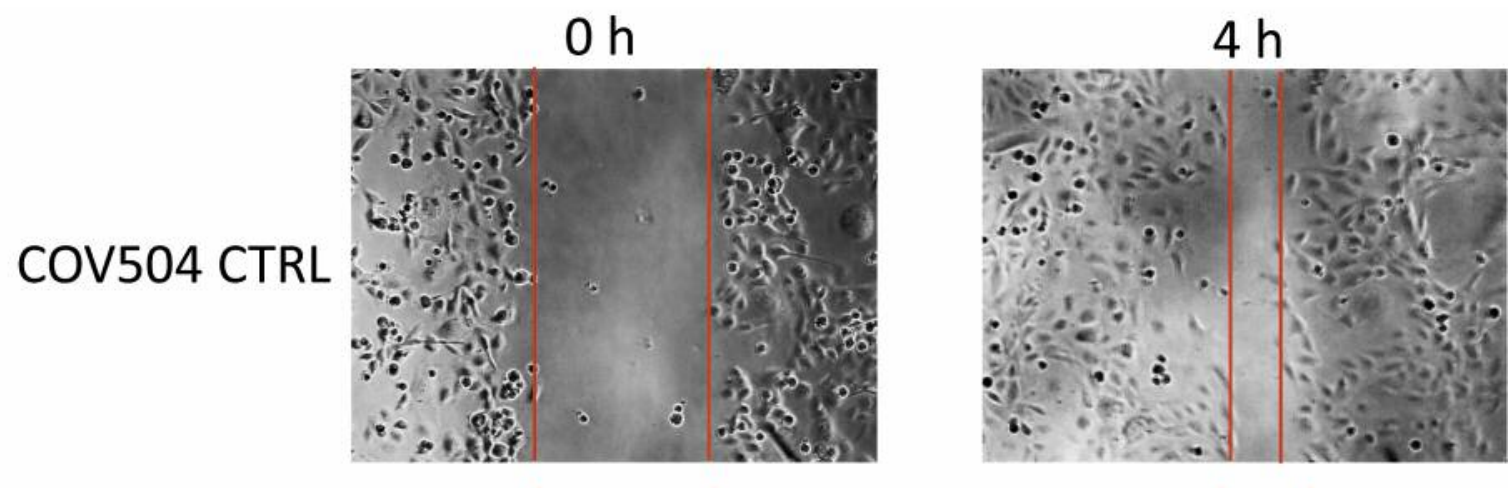

COV504 KD
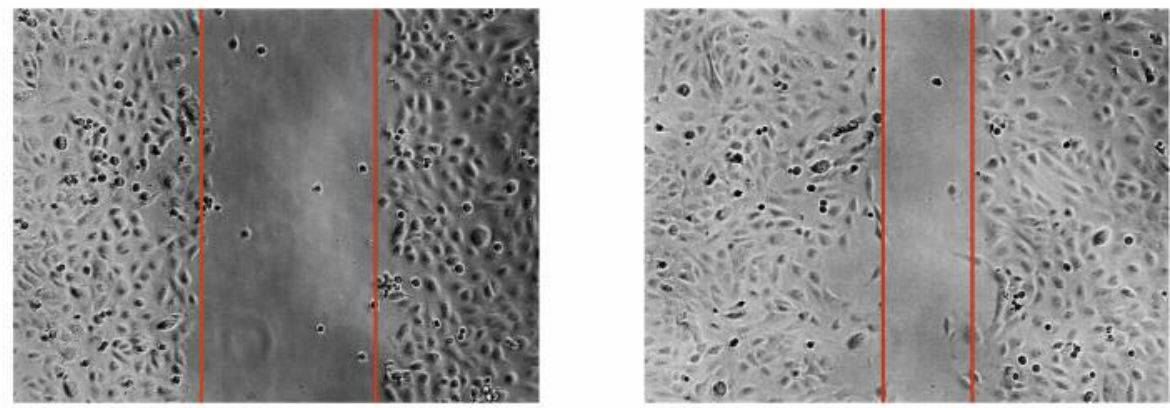

SKOV3 CTRL
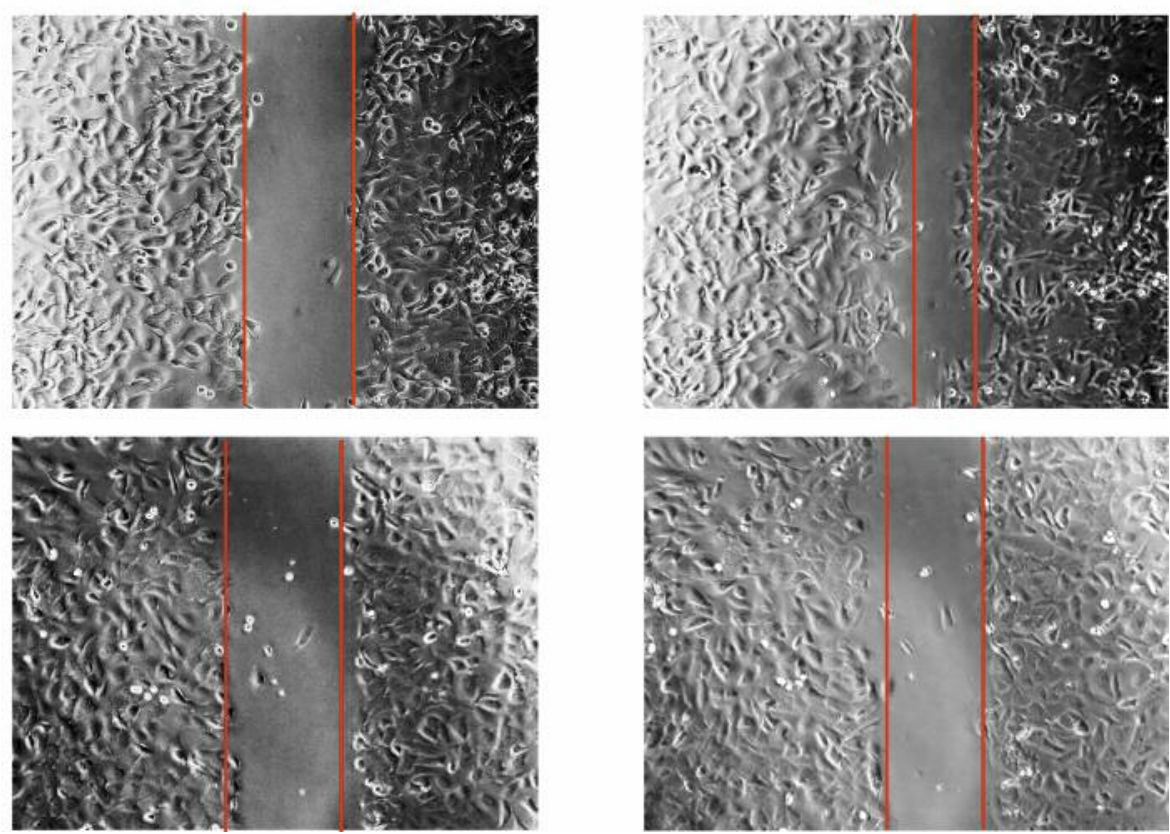

Figure 4. Scratch wound healing showing the reduction of the migration capacity in the OC cells with EPHAl knockdown. The monolayer of cultured cells was scratched using a pipette tip to form a gap. The gap closure by cancer cells, which indicates cancer cell migration, was captured under an inverted microscopy after cultivation for $4 \mathrm{~h}$. The red lines highlight the remaining scratch area. CTRL, Control; KD, knockdown; OC, ovarian cancer.

Knockdown of EPHAl reduced cellular adhesion of OC cells to Matrigel. As shown in Figure 3, KD of EPHAl in COV504 cells decreased the adherent capacity to approximately $24 \%$ compared to the control cells $(p<0.05)$. Likewise, KD of EPHA1 in SKOV3 cells also decreased the cellular adhesion capacity to approximately $40 \%$
Knockdown of EPHAl inhibited migration capacity of OC cells. We evaluated the migration capacity of the OC cells using the scratch wound-healing assay. EPHAl KD in COV504 cells decreased the rate of the wound healing from $70.13 \%$ to $44.62 \% 4$ hours after scratch wounding. Likewise, EPHA1 KD in SKOV3 cells decreased the rate of wound 
A

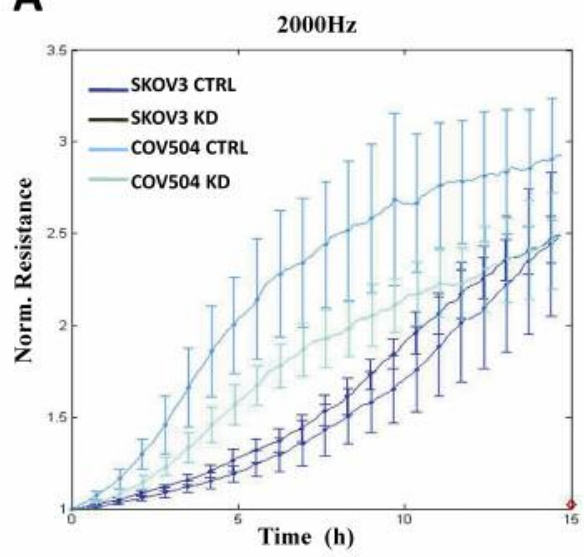

B

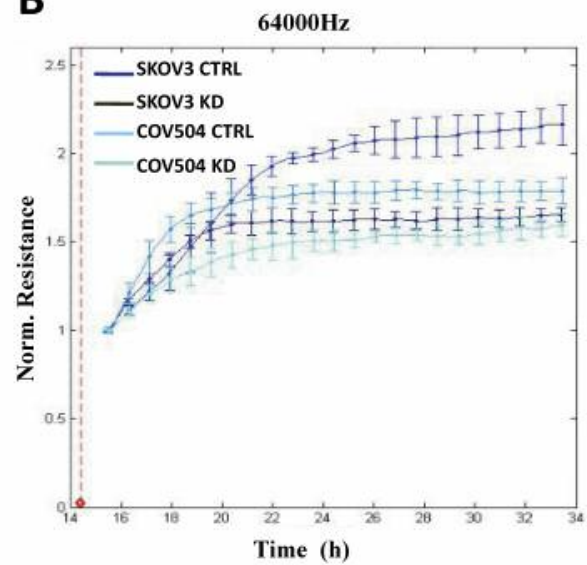

Figure 5. Initial cell spreading/attachment and post-wound migration capacity of the OC cells indicated in the Electric Cell-substrate Impedance Sensing (ECIS) system. (A) The normalized resistance of the cells at 2,000 Hz for the first $15 \mathrm{~h}$ indicating the cellular property of spreading and attachment. (B) Following an electric wound $(30 \mathrm{~s}, 2,600 \mathrm{~Hz})$, the normalized resistance of the cells at 64,000 $\mathrm{Hz}$ indicating the dynamic change of migration of the OC cells. CTRL, Control; KD, knockdown; OC, ovarian cancer.

A

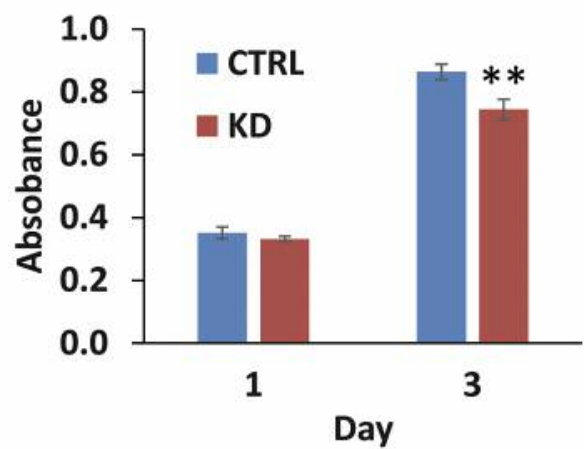

C

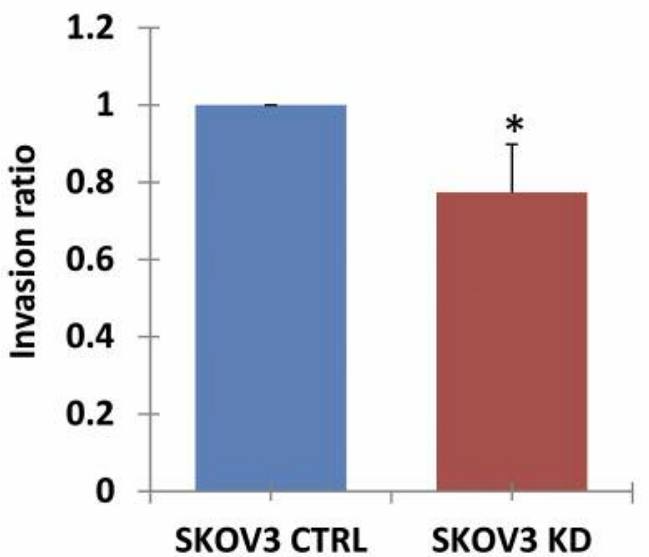

B

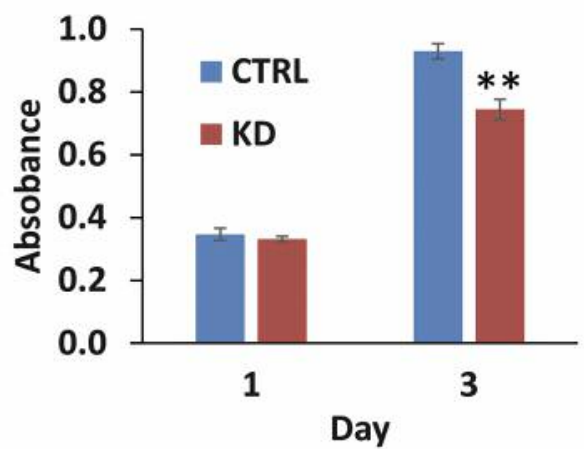

D

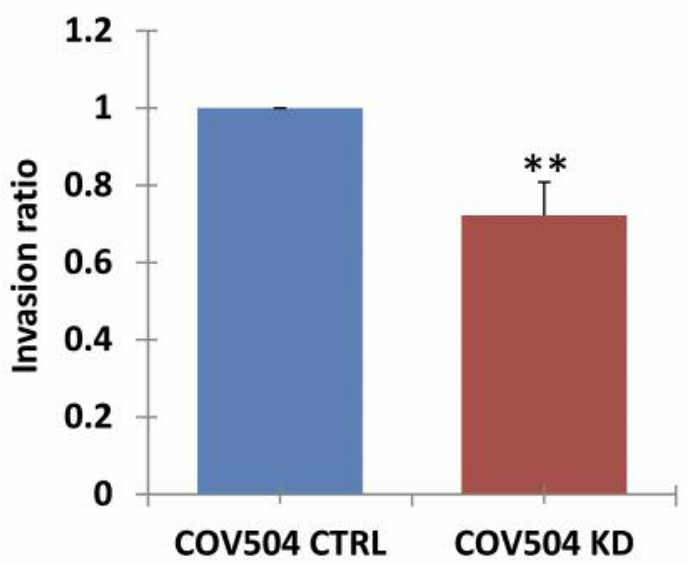

Figure 6. Evaluation of proliferation and invasion of OC cells after EPHA1 knockdown. (A) Crystal violet proliferation assay. (B) Transwell invasion property of the $O C$ cells normalized using the control. The transwell inserts with $8 \mu \mathrm{m}$ pore size were coated with Matrigel gel first to mimic the extracellular matrix in a tumor microenvironment. Data are presented as means with standard deviation (SD) (triple test, $n=3)$. * $p<0.05$. **p<0.01. CTRL, Control; KD, knockdown; OC, ovarian cancer. 


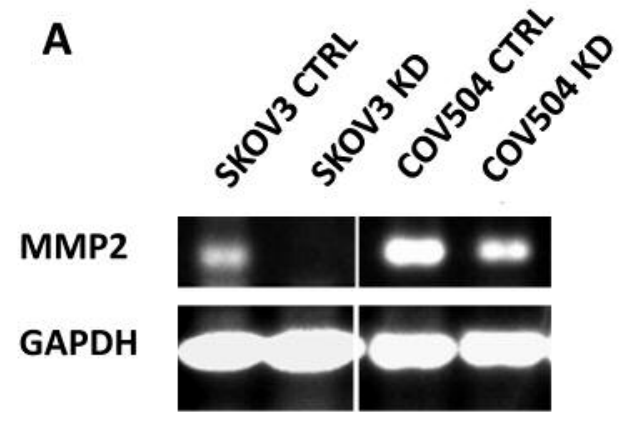

C

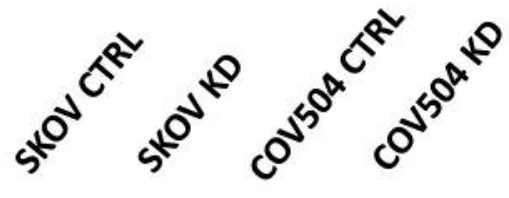

B B Gelatin zymography:MMP2

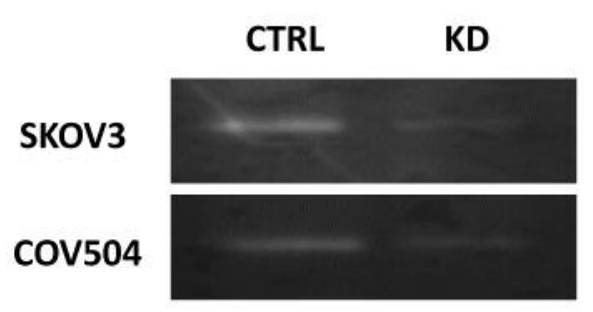

D

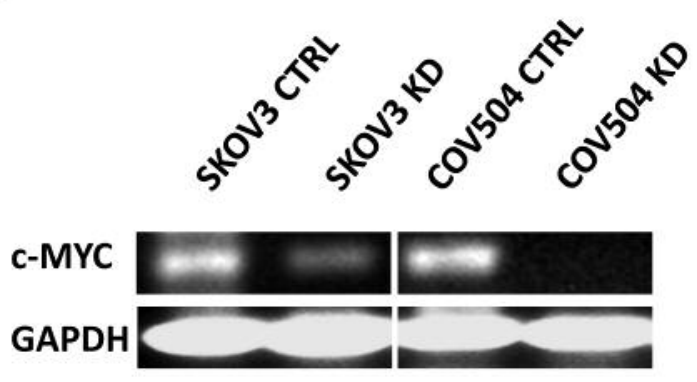

Figure 7. Identification of key signalling pathway proteins after EPHA1 knockdown in OC cells. (A) Western blotting showing the down-regulation of MMP2 protein after EPHA1 knockdown. (B) Examination of the matrix cleavage activity of MMP2 (gelatinase) using the gelatin zymography assay. (C) IP of ERK after pulling down of whole cell lysate proteins using a phospho-tyrosine mouse mAb (P-Tyr) (top) in comparison to the western blotting of ERK1/2 without IP (bottom). (D) Western blotting of c-MYC in whole cell lysates. IB, Immunoblotting; IP, immunoprecipitation; MMP2, metalloproteinase-2; ERK, extracellular signal-regulated kinase; CTRL, control; KD, knockdown; OC, ovarian cancer; mAb, monoclonal antibody.

healing from $68.75 \%$ to $49.23 \% 4$ hours after scratch (Figure 4). Using the ECIS system, we were able to quantify the attachment and post-wound migration of cells in a highthroughput real-time manner. As shown in Figure 5, The ECIS data at 2,000 Hz indicated that the COV504 cells with EPHAl KD had a lower level of attachment than the control to the gold electrode $(p<0.05)$. However, the SKOV3 cells with EPHA1 KD did not show significant difference (Figure $5 \mathrm{~A}$ ). Following electric wound in the ECIS system (Figure 5B), both SKOV3 and COV504 cells with EPHA1 KD showed suppressed level of migration (at 64,000 Hz) compared to their control cells after electrical wounding, respectively $(p<0.01)$, supporting the observations from the scratch wounding assays.

Knockdown of EPHAl inhibited proliferation and transwell invasion of $O C$ cells. The data from the crystal violet proliferation assay indicated that SKOV3 cells with EPHAl KD decreased the proliferation of cells by approximately $13.79 \%$ after culture for 3 days compared to the control $(p<0.01$; Figure 6A). We also observed an approximately
$19.44 \%$ decrease of proliferation from the stable COV504 cells with EPHA1 KD compared to the backbone control $(p<0.01)$. The result from the transwell inset invasion assay showed that EPHAl KD, in SKOV3 cells, led to a decrease in invasion by approximately $22.56 \%$ ( $p<0.05 v s$. control). Similarly, EPHA1 KD, in COV504 cells, led to a decrease of invasion by approximately $27.80 \%$ compared to the control $(p<0.01$ vs. control).

Knockdown of EPHAl inhibited the activity of MMP-2 and cMYC signaling pathways. We further investigated the possible mechanism that EPHAl KD had an effect on the behavior of the OC cells. The Western blotting data showed that MMP2 protein expression was down-regulated following EPHA1 KD in both SKOV3 and COV504 cells (Figure 7A). The inhibition of MMP2 activity was also confirmed using gelatin zymography in the two cell types (Figure 7B). It appears that the interaction of P-TY and ERK was inhibited by EPHAl knockdown. Both ERK1 and ERK2 proteins were upregulated by EPHA1 KD in SKOV3 cells. However, in COV504 cells with EPHAl KD, ERK1 was down-regulated, 
but ERK2 was up-regulated (Figure 7C). By western blotting, we also found that the c-MYC protein expression was downregulated after EPHAl $\mathrm{KD}$ in both cell types and the reduction was more distinctive in COV504 cells (Figure 7D).

\section{Discussion}

Previous studies suggest that EPHA1 may be implicated in the prognosis and progression of various cancers with a diverse manner. For example, EPHA1 is positively associated with tumor proliferative capacity in non-small cell lung carcinoma (22), whilst, in gastric cancer, the EPHA1 transcript expression level appears to be positively associated with tumor size, stage and lymph node metastasis (23). Also, in pancreatic ductal adenocarcinoma, EPHA1 intensity is significantly associated with tumor size and histopathological stage as indicated by immunohistochemical staining (24). However, in clear cell renal cell carcinoma, positive EPHA1 protein staining, as well as a low Ephrin A1 protein levels, are significantly linked to more aggressive tumor features and poor survival (25). In prostate cancer, increased level of EPHA1 protein is frequently correlated with a high Gleason score (16). In contrast, in colorectal cancer (CRC), down-regulation or loss of EPHA1 expression, probably due to DNA hypermethylation, appears to be correlated with invasion, metastasis and poor survival of patients depending on stages $(26,27)$. However, in OC, the association of EPHA 1 with cancer stage is not conclusive. It is reported that increased levels of Ephrin A1, the preferential ligand for EPHA1 and a high-affinity ligand for EphA2, is strongly correlated with poor survival, suggesting that altered Ephrin A1/EPHA1 signaling pathway may be involved in the aggression of this malignancy.

We, herein, took advantage of the potent CRISPR/Cas9 genome editing technique to KD EPHAl specifically (28, 29). In the stable OC cells with EPHAl KD, there was accumulative arrest of $\mathrm{G}_{1}$ and/or sub- $\mathrm{G}_{1}$ with corresponding decrease of $M$ phase subsets. There is evidence that the activation of EPHA1 through Ephrin may inhibit ERK, which mediates multiple cancer cell behaviors, including cell cycle phases in a P53-indepentent manner $(30,31)$. We further investigated the change of ERK1 and ERK2 protein isoforms. It appears that KD of EPHAl in SKOV3 cells increases the protein levels of both ERK1 and ERK2. However, in COV504, EPHAl KD increases the level of ERK2 but decreases ERK1. After phospho-tyrosine proteins are pulled down using a P-TYR monoclonal antibody (P-Tyr) by immunoprecipitation, there is no change of ERK, implying its transcriptional repressor role is independent of its kinase activity (32).

We also observed that EPHAI KD inhibits the adhesion ability of both OC cell lines tested. One previous study shows that there is interaction between EPHA1 and integrinlinked kinase, which mediates cellular spreading and adhesion to extracellular matrix depending on cell types (33).

We observed that KD of EPHAl down-regulates c-MYC expression in OC cells. C-MYC is a well-characterized proto-oncogene that has frequently been found to be constitutively activated in various cancer types, including OC (34). As a transcription factor, C-MYC exerts diverse regulatory functions in cancer cells, including cell cycle, cell adhesion, cellular metabolism and apoptotic pathways (35). Therefore, the suppressed aggressive capacity of the OC cells after EPHAI KD may also attribute to decreased expression of c-MYC.

Our data from the ECIS system further confirm that EPHA1 KD not only inhibited cellular spreading but also post-wound migration of the OC cells. The inhibited invasion by EPHAI KD may be attributed to the downregulation of MMP2, as shown by western blotting and zymography. This finding is partially supported by previous study in which EPHAl silencing causes down-regulation of MMP2 in hepatocellular carcinoma cells (36).

Taken together, our data showed that EPHAl KD suppresses the malignant properties of the OC cells in different aspects, which include cell cycle arrest, cell adhesion migration, proliferation and invasion. These findings enable us to propose that overexpression or activation of EPHAI may promote aggression of OC. Therefore EPHA1 may possess potential therapeutic value as an anticancer target in OC patients with EPHA1 or Ephrin A1 overexpression.

\section{Conflicts of Interest}

The Authors declare no conflicts of interest.

\section{Acknowledgements}

The Authors thank the support from CCMRC and Cancer Research Wales. They also thank Fiona Ruge for her technical assistance.

\section{References}

1 Ferlay J, Shin HR, Bray F, Forman D, Mathers C and Parkin DM: Estimates of worldwide burden of cancer in 2008: GLOBOCAN 2008. Int J Cancer 127: 2893-2917, 2010.

2 Ferlay J, Soerjomataram I, Dikshit R, Eser S, Mathers C, Rebelo M, Parkin DM, Forman D and Bray F: Cancer incidence and mortality worldwide: Sources, methods and major patterns in GLOBOCAN 2012. Int J Cancer 136: E359-E386, 2015.

3 Siegel RL, Miller KD and Jemal A: Cancer statistics, 2016. CA Cancer J Clin 66: 7-30, 2016.

4 El Bairi K, Kandhro AH, Gouri A, Mahfoud W, Louanjli N, Saadani B, Afqir S and Amrani M: Emerging diagnostic, prognostic and therapeutic biomarkers for ovarian cancer. Cell Oncol (Dordr) 40(2): 105-118, 2017. 
5 Schüler S, Ponnath M, Engel J and Ortmann O: Ovarian epithelial tumors and reproductive factors: A systematic review. Arch Gynecol Obstet 287: 1187-1204, 2013.

6 Holschneider $\mathrm{CH}$ and Berek JS: Ovarian cancer: epidemiology, biology and prognostic factors. In: Semin Surg Oncol: Wiley Online Library, pp. 3-10, 2000.

7 Perez White BE and Getsios S: Eph receptor and ephrin function in breast, gut and skin epithelia. Cell Adh Migr 8: 327-338, 2014.

8 Shiuan E and Chen J: Eph receptor tyrosine kinases in tumor immunity. Cancer Res 76: 6452-6457, 2016.

9 Wei Q, Liu J, Wang N, Zhang X, Jin J, Chin-Sang I, Zheng J and Jia Z: Structures of an Eph receptor tyrosine kinase and its potential activation mechanism. Acta Crystallogr D Biol Crystallogr 70: 3135-3143, 2014.

10 Kania A and Klein R: Mechanisms of ephrin-Eph signalling in development, physiology and disease. Nat Rev Mol Cell Biol 17: 240-256, 2016.

11 Shiuan E and Chen J: Eph receptor tyrosine kinases in tumor immunity. Cancer Res 76: 6452-6457, 2016.

12 Gucciardo E, Sugiyama N and Lehti K: Eph- and ephrindependent mechanisms in tumor and stem cell dynamics. Cell Mol Life Sci 71: 3685-3710, 2014.

13 Hirai H, Maru Y, Hagiwara K, Nishida J and Takaku F: A novel putative tyrosine kinase receptor encoded by the eph gene. Science 238: 1717-1720, 1987.

14 Herath NI, Spanevello MD, Doecke JD, Smith FM, Pouponnot $\mathrm{C}$ and Boyd AW: Complex expression patterns of Eph receptor tyrosine kinases and their ephrin ligands in colorectal carcinogenesis. Eur J Cancer 48: 753-762, 2012.

15 Fox BP and Kandpal RP: Invasiveness of breast carcinoma cells and transcript profile: Eph receptors and ephrin ligands as molecular markers of potential diagnostic and prognostic application. Biochem Biophys Res Commun 318: 882-892, 2004.

16 Peng L, Wang H, Dong Y, Ma J, Wen J, Wu J, Wang X, Zhou X and Wang J: Increased expression of EphA1 protein in prostate cancers correlates with high Gleason score. Int J Clin Exp Pathol 6: 1854-1860, 2013.

17 Hafner C, Becker B, Landthaler $M$ and Vogt T: Expression profile of Eph receptors and ephrin ligands in human skin and down-regulation of EphA1 in nonmelanoma skin cancer. Mod Pathol 19: 1369-1377, 2006.

18 Herath NI, Spanevello MD, Sabesan S, Newton T, Cummings M, Duffy S, Lincoln D, Boyle G, Parsons PG and Boyd AW: Over-expression of Eph and ephrin genes in advanced ovarian cancer: Ephrin gene expression correlates with shortened survival. BMC Cancer 6: 144, 2006.

19 Wu BO, Jiang WG, Zhou D and Cui YX: Knockdown of EPHA1 by CRISPR/CAS9 promotes adhesion and motility of HRT18 colorectal carcinoma cells. Anticancer Res 36: 1211-1219, 2016.

20 Jiang WG, Ye L, Sanders AJ, Ruge F, Kynaston HG, Ablin RJ and Mason MD: Prostate transglutaminase (TGase-4, TGaseP) enhances the adhesion of prostate cancer cells to extracellular matrix, the potential role of TGase-core domain. J Transl Med 11: 269, 2013.

21 Kaplan EL and Meier P: Nonparametric estimation from incomplete observations. J Am Stat Assoc 53: 457-481, 1958.

22 Giaginis C, Tsoukalas N, Bournakis E, Alexandrou P, Kavantzas N, Patsouris E and Theocharis S: Ephrin (Eph) receptor A1, A4, A5 and A7 expression in human non-small cell lung carcinoma: associations with clinicopathological parameters, tumor proliferative capacity and patients' survival. BMC Clin Pathol 14: 8, 2014.
23 Wang J, Dong Y, Wang X, Ma H, Sheng Z, Li G, Lu G, Sugimura $\mathrm{H}$ and Zhou $\mathrm{X}$ : Expression of EphA1 in gastric carcinomas is associated with metastasis and survival. Oncol Rep 24: 1577, 2010.

24 Giaginis C, Tsourouflis G, Zizi-Serbetzoglou A, Kouraklis G, Chatzopoulou E, Dimakopoulou K and Theocharis SE: Clinical significance of ephrin (eph)-A1,-A2,-a4,-a5 and-a7 receptors in pancreatic ductal adenocarcinoma. Pathol Oncol Res 16: 267-276, 2010.

25 Toma MI, Erdmann K, Diezel M, Meinhardt M, Zastrow S, Fuessel S, Wirth MP and Baretton GB: Lack of ephrin receptor A1 is a favorable independent prognostic factor in clear cell renal cell carcinoma. PloS One 9: e102262, 2014.

26 Herath N, Doecke J, Spanevello M, Leggett B and Boyd A: Epigenetic silencing of EphA1 expression in colorectal cancer is correlated with poor survival. Br J Cancer 100: 1095-1102, 2009.

27 Dong Y, Wang J, Sheng Z, Li G, Ma H, Wang X, Zhang R, Lu $\mathrm{G}, \mathrm{Hu} \mathrm{Q}$ and Sugimura H: Down-regulation of EphA1 in colorectal carcinomas correlates with invasion and metastasis. Mod Pathol 22: 151-160, 2009.

28 Ran FA, Hsu PD, Wright J, Agarwala V, Scott DA and Zhang F: Genome engineering using the CRISPR-Cas9 system. Nat Protoc 8: 2281-2308, 2013

29 Perez-Pinera P, Kocak DD, Vockley CM, Adler AF, Kabadi AM, Polstein LR, Thakore PI, Glass KA, Ousterout DG and Leong KW: RNA-guided gene activation by CRISPR-Cas9-based transcription factors. Nat Methods 10: 973-976, 2013.

30 Chambard J-C, Lefloch R, Pouysségur J and Lenormand P: ERK implication in cell cycle regulation. Biochim Biophys Acta 1773: 1299-1310, 2007.

31 Yamamoto T, Ebisuya M, Ashida F, Okamoto K, Yonehara S and Nishida E: Continuous ERK activation down-regulates antiproliferative genes throughout $\mathrm{G}_{1}$ phase to allow cell-cycle progression. Curr Biol 16: 1171-1182, 2006.

32 Whitmarsh AJ: Regulation of gene transcription by mitogenactivated protein kinase signaling pathways. Biochim Biophys Acta 1773: 1285-1298, 2007.

33 Yamazaki T, Masuda J, Omori T, Usui R, Akiyama H and Maru Y: EphA1 interacts with integrin-linked kinase and regulates cell morphology and motility. J Cell Sci 122: 243-255, 2009.

34 Jung M, Russell AJ, Liu B, George J, Liu PY, Liu T, DeFazio A, Bowtell DD, Oberthuer A, London WB, Fletcher JI, Haber M, Norris MD and Henderson MJ: A myc activity signature predicts poor clinical outcomes in myc-associated cancers. Cancer Res 77: 971-981, 2017.

35 Dang CV: MYC on the path to cancer. Cell 149: 22-35, 2012.

36 Chen G, Wang Y, Zhou M, Shi H, Yu Z, Zhu Y and Yu F: EphA1 receptor silencing by small interfering RNA has antiangiogenic and antitumor efficacy in hepatocellular carcinoma. Oncol Rep 23: $563,2010$. 